\title{
Identification of a rare case of intra-articular osteochondroma manifesting as three loose bodies in a patient with hereditary multiple osteochondromas: A case report
}

\author{
MINGXIANG KONG ${ }^{1}$, LI CAO $^{1}$, QIONG ZHANG ${ }^{2}$, YONG FANG $^{1}, \mathrm{CHEN} \mathrm{ZHAO}^{1}$, \\ HAIFENG GU $^{1}$, SHUIJUN ZHANG ${ }^{1}$, YU CHEN ${ }^{1}$, JINHUA WU ${ }^{1}$ and QING BI ${ }^{1}$ \\ ${ }^{1}$ Department of Orthopedics and Joint Surgery; \\ ${ }^{2}$ Operating Room, Zhejiang Provincial People's Hospital, Hangzhou, Zhejiang 310014, P.R. China
}

Received September 25, 2014; Accepted May 13, 2015

DOI: $10.3892 / \mathrm{ol} .2015 .3284$

\begin{abstract}
Hereditary multiple osteochondromas (HMO) is an autosomal dominant bone disorder characterised by the presence of multiple benign cartilage-capped tumours. Exostosin-1 (EXT1) and EXT2 are the major morbigenous genes associated with HMO, mutations in which are responsible for 90\% of all HMO cases. In patients with HMO, osteochondromas arise adjacent to the metaphysis and typically remain in the metaphyseal region of the long bones. Therefore, it is rare for osteochondromas to be identified intra-articularly, although they may manifest as loose bodies. The present study describes a rare case of HMO manifesting as limited flexing range in the right knee joint of a 27-year-old male patient. Computed tomography and magnetic resonance imaging (MRI) revealed three intra-articular osteochondromas located in the intercondylar fossa of the patient's right knee. The intra-articular osteochondromas and protuberant extra-articular osteochondromas around the right knee were resected, resulting in improved right knee function and no postoperative recurrence. Pathological analysis revealed that the intra-articular osteochondromas had a thinner cartilage cap layer than the extra-articular osteochondromas. In addition, genetic analysis of the patient and the patient's mother was conducted. From this, it was determined that a nonsense mutation, c.115G>T (p.E39X) in exon 1 of the EXT1 gene, was the cause of HMO in this case. Thus, it is proposed that osteochondromas with a pedicle within the knee, may tear and become loose intra-articular bodies, resulting in limited joint function and thereby contributing to the progression of HMO.
\end{abstract}

Correspondence to: Professor Qing Bi, Department of Orthopedics and Joint Surgery, Zhejiang Provincial People's Hospital, 158 Shangtang Road, Hangzhou, Zhejiang 310014, P.R. China E-mail: 13588302991@126.com

Key words: hereditary multiple osteochondromas, exostosin-1 gene, intra-articular, nonsense mutation, loose body

\section{Introduction}

Osteochondromas, also termed osteocartilaginous exostoses, comprise the majority of bone tumours in the human skeleton. These tumours are benign osseous growths that are capped with hyaline cartilage (1). Solitary osteochondromas develop in a single bone and are not hereditary. By contrast, multiple osteochondromas, also known as hereditary multiple osteochondromas (HMO), result from an autosomal dominant disorder characterised by the abundant proliferation of exostoses (2). Genetic studies have identified an association between HMO and three loci: i) Exostosin-1 (EXT1) (3), which maps to chromosome 8q24.1; ii) EXT2 (4), which maps to chromosome 11p13; and iii) EXT3 (5), which is located on the short arm of chromosome 19 (the exact position has yet to be mapped). It has been estimated that half of all patients with HMO have EXT1 mutations, while one-third have EXT2 mutations (6).

Multiple osteochondromas typically increase in size and number during childhood and adolescence. Although these tumours primarily develop at the juxta-epiphyseal region of the long bones, they can occur on nearly every bone of the skeleton, including short bones, flat bones and irregular bones (7). Patients with HMO are generally asymptomatic, unless the osteochondroma exerts pressure on adjacent muscles, tendons, nerves or blood vessels (8). The clinical presentation is commonly associated with this effect, and includes pain, angular deformities, short stature, restricted joint motion, fractures of the lesion itself, inflammatory changes of the bursa exostotica covering the cartilage cap and malignant transformation (9).

Osteochondromas typically occur around the growth plate of long bones in childhood and then move toward the diaphysis as growth occurs (10). Therefore, osteochondromas are generally located extra-articularly. Intra-articular osteochondromas are rare, but do occur, and may cause pain and discomfort, as well as range-of-motion restrictions.

The present study investigated the clinical, imaging, histological and genetic aspects of a 27 -year-old male patient with typical HMO who presented with intra-articular osteochondromas, manifesting as bony loose bodies within the knee joint. To the best of our knowledge, the present case is the first reported incidence of intra-articular osteochondromas 
manifesting as $>1$ loose body in the joint of a patient with HMO. In addition, the current study performed genetic analysis of the patient and the patient's family members with the aim of identifying the underlying mutation causing HMO in this family.

\section{Case report}

A 27-year-old male patient of short stature, presented to Zhejiang Provincial People's Hospital (Hangzhou, China) on May 5th, 2013, with multiple elevated bony prominences and a limited ability to bend the right knee, which had been ongoing for 10 years. No history of trauma was reported by the patient. The bony prominences and surface anomalies were asymptomatic, and initially arose when the patient was 6 years old; the bony prominences had progressively increased in size and number since, with pain initially experienced 10 years previously, on maximal flexion of the knee. The patient progressively experienced limitation in squatting ability. He was also was uncomfortable with the appearance of the multiple osteochondromas.

The patient had a family history of HMO on the maternal side. The patient's mother was also of short stature and had multiple elevated exostoses in various locations, although no limitations in joint mobility. The patient's maternal grandmother and uncle had also been diagnosed with HMO. However, these two family members had succumbed to unknown causes a number of years previously.

Physical examination revealed multiple bony prominences around the knees and no significant symptoms at rest. However, there was significant pain in the right knee joint during loaded flexion. Although the patient was capable of full extension, maximum flexion was $85^{\circ}$. The collateral and cruciate ligaments were stable, and the medial joint line and medial femoral condyle were tender to palpation. The function of his left knee was normal. Plain radiographs demonstrated an intra-articular, mineralised mass at the intercondylar fossa (Fig. 1). Computed tomography $(\mathrm{CT})$ and three-dimensional CT reconstruction of the right knee revealed three mineralised masses manifesting as loose bodies at the intercondylar fossa and no abnormalities in other intra-articular structures (Fig. 2). In addition, magnetic resonance imaging (MRI) revealed that the anterior mass was located in close proximity to the posterior cruciate ligament (PCL; Fig. 3).

Due to the pain and limited range of motion experienced by the patient, as well as limited knowledge of the exact nature of the lesion, an arthroscopic excision was performed. The knee was passively flexed under general anaesthesia. A decreased range of flexion with maximum flexion of $85^{\circ}$, was observed. Arthroscopic standard portals were created and the intercondylar view revealed an oval mass with a cartilaginous surface between the concave condyles. The mass was located posterior to the anterior cruciate ligament (ACL) and adjacent to the PCL. The mass filled the majority of the intercondylar fossa space and mild synovial joint hyperplasia was observed. The ligaments, cartilage and menisci were intact, and patellar tracking was normal. A probe demonstrated that the mass had limited mobility, indicating that the mass was a loose body (Fig. 4A). The lesions was then removed, and a further small mass was observed,

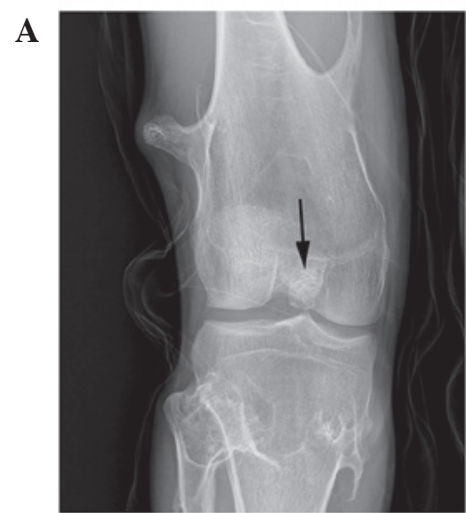

$\mathbf{B}$

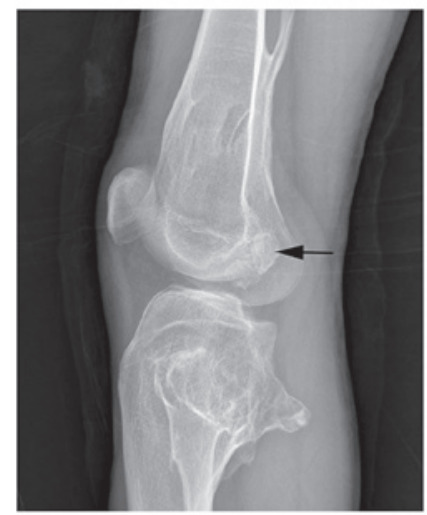

Figure 1. Plain radiographs demonstrating an osteochondroma located at the intercondylar fossa in the patient's right knee (arrows). (A) Anteroposterior and (B) lateral films. In addition, other extra-articular osteochondromas growing toward the diaphysis were observed.

just posterior to the first (Fig. 4B). This small mass was also removed and a third large mass was observed posterior to the PCL (Fig. 4C). The two posterior masses also appeared to be loose bodies. The third mass was removed and the intercondylar fossa was free of bony tissue (Fig. 4D). The knee was bent again and a dramatic improvement in flexion was observed (maximum flexion, $110^{\circ}$ ), demonstrating that the intra-articular masses had been preventing the right knee from fully flexing. Macroscopically, the three excised masses measured $2.0 \times 1.5 \times 1.5 \mathrm{~cm}, 1.0 \times 1.0 \times 0.5 \mathrm{~cm}$ and $3.0 \times 3.0 \times 2.0 \mathrm{~cm}$, respectively (Fig. 5A). For aesthetic reasons, the protuberant extra-articular osteochondromas in the tibia and femur around the right knee were also resected with small incisions. Pathological analysis under the microscope (BX50; Olympus Corporation, Tokyo, Japan) demonstrated that the extra-articular masses were typical osteochondromas, which were composed of three layers (fibrous perichondrium, cartilage cap and bone; Fig. 5D). However, in contrast to the extra-articular osteochondromas, pathological examination of the intra-articular osteochondromas revealed thinner layers of fibrous perichondrium and cartilage cap, and a thicker layer of bone (3.6 vs. 2.0-mm thick cartilage; Fig. 5C). Following arthroscopy, the pain previously experienced during loaded flexion in the right knee disappeared and the range of motion recovered to $120^{\circ}$ degrees. Imaging confirmed that the three masses had been removed (Fig. 6). One year after removal, the patient was asymptomatic with respect to his knee, with no signs of recurrence evident on radiographic analysis. 
A

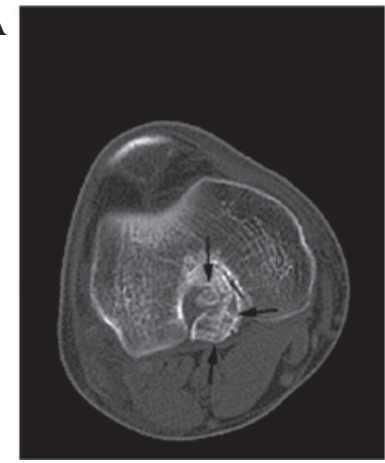

B

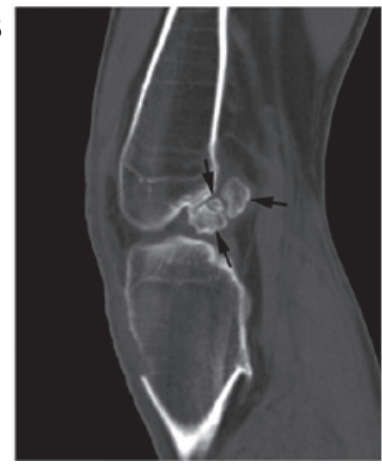

C

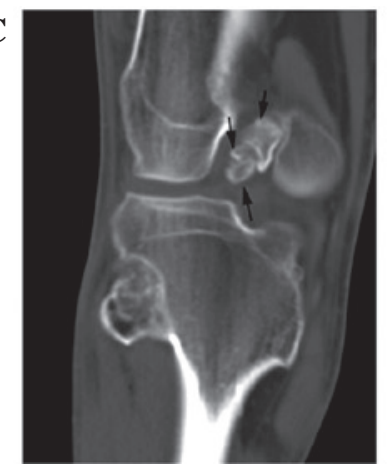

D

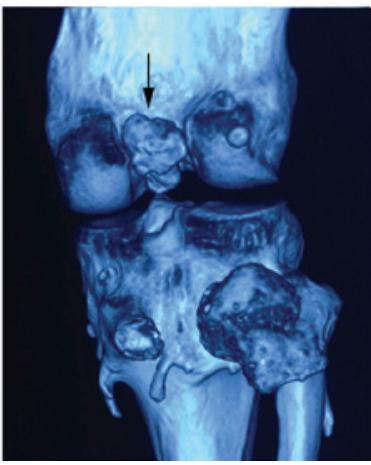

Figure 2. Preoperative (A-C) CT and (D) 3D CT reconstruction of the right knee, revealing three osteochondromas on the intercondylar fossa of the knee, which manifested as loose bodies that were not attached to the femur. The 3D CT reconstruction indicated that the bony loose bodies were located in an area that would impact the posterior contour of the medial condyle of the tibia upon flexion. CT, computed tomography.

A

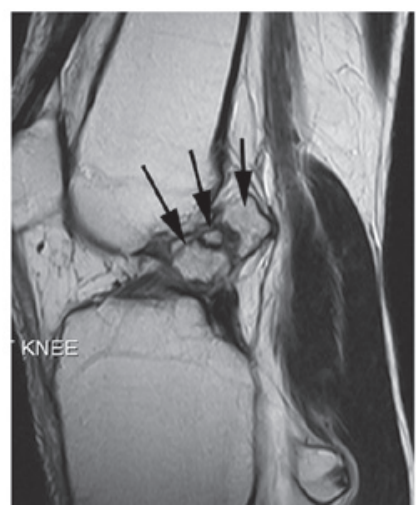

B

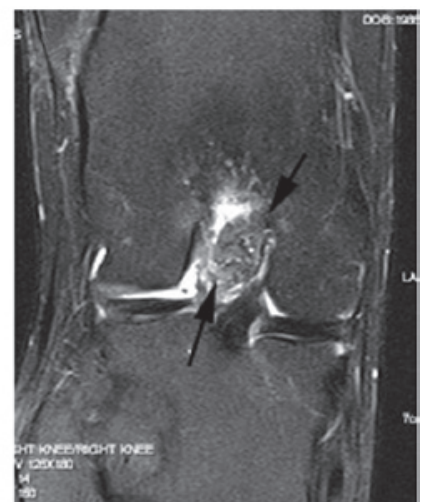

Figure 3. Preoperative magnetic resonance imaging of the right knee, revealing three well-demarcated inhomogeneous lesions, with mixed high and low signals on T2-weighted imaging in the intercondylar fossa of the knee. (A) Coronal and (B) sagittal images. Arrows indicate the three osteochondromas. The anterior mass was located in close proximity to the posterior cruciate ligament.

A

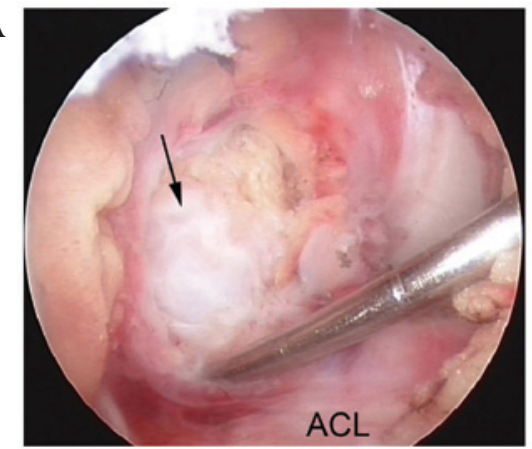

$\mathbf{C}$

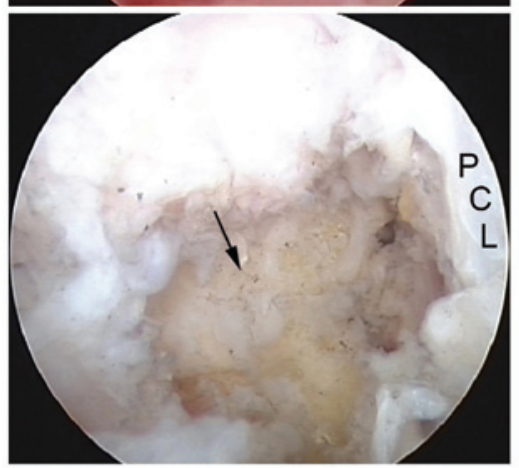

B

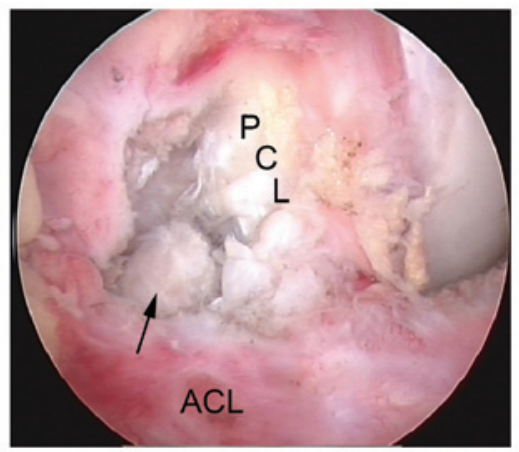

D

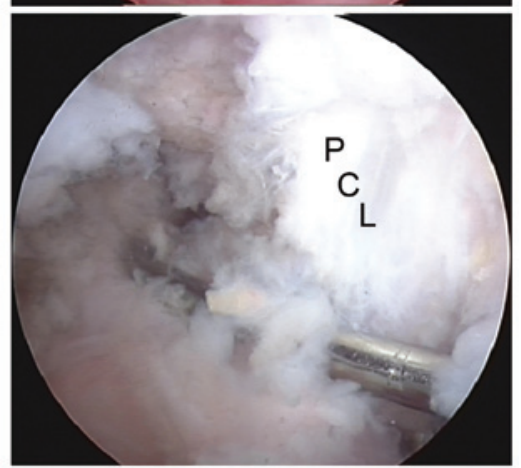

Figure 4. Arthroscopic view of the intercondylar fossa of the knee joint, revealing mild synovial hyperplasia of the joint. Arrows indicate osteochondromas. The first bony mass was covered by a layer of connective tissue. After the connective tissue was incised, white-coloured cartilage was revealed. (A) A probe demonstrated that the mass had limited mobility, indicating that the mass was a loose body. (B) The first mass was removed revealing a smaller mass in the intercondylar fossa. (C) The second mass was removed revealing the largest mass deep in the intercondylar fossa. (D) The third mass was also removed leaving the intercondylar fossa free of bony tissues. ACL, anterior cruciate ligament; PCL, posterior cruciate ligament. 
A
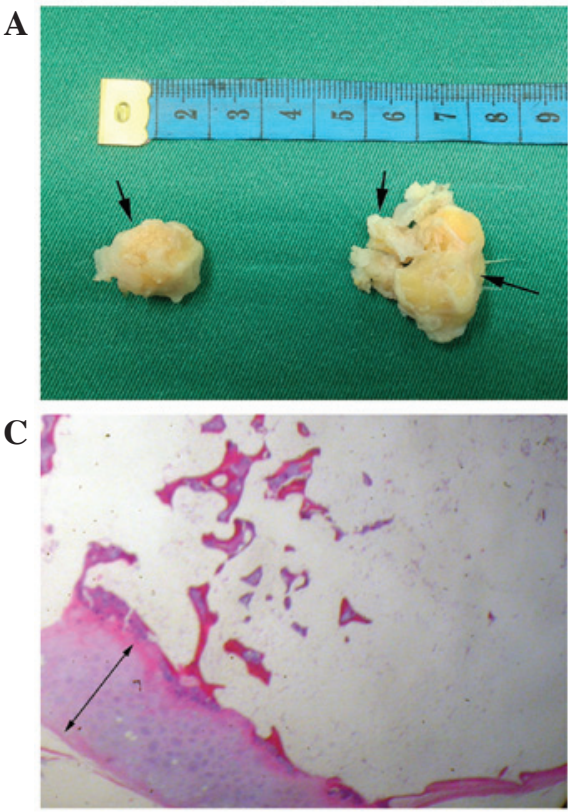

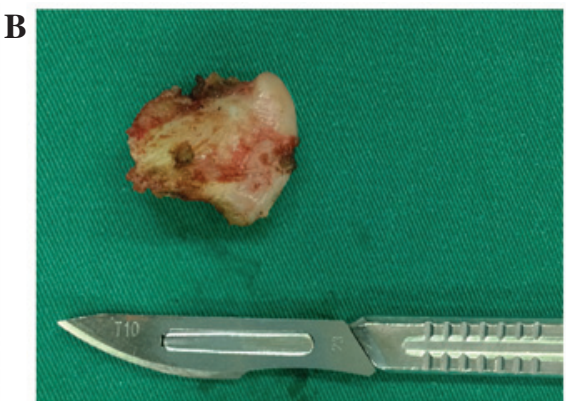

D

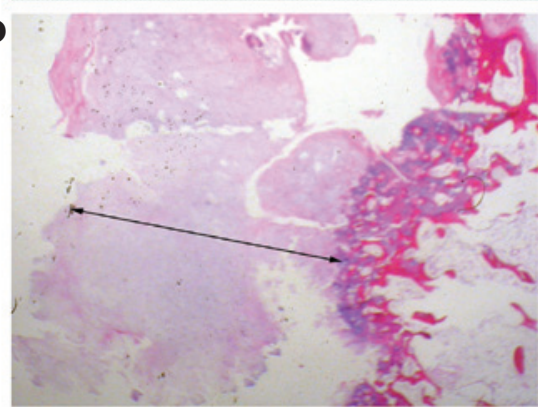

Figure 5. Gross structure and histological view of the masses. Gross structure of (A) the three intra-articular masses (arrows) and (B) one of the extra-articular masses excised from around the knee. (C) and (D) Histopathological analysis of the lesions demonstrating that the osteochondromas had three layers (the perichondrium, cartilage cap and bone). The cartilage cap is stained pale blue (double-headed arrows) and the underlying bone is stained pink (hematoxylin and eosin staining; magnification, x20). Compared with (D) the extra-articular osteochondroma, the histopathology of (C) the intra-articular osteochondroma appeared to have thinner layers of the fibrous perichondrium and cartilage cap, as well as a thicker layer of bone. The mean cartilage thickness was 2.0 mm in the intra-articular osteochondroma and $3.6 \mathrm{~mm}$ in the extra-articular osteochondroma.
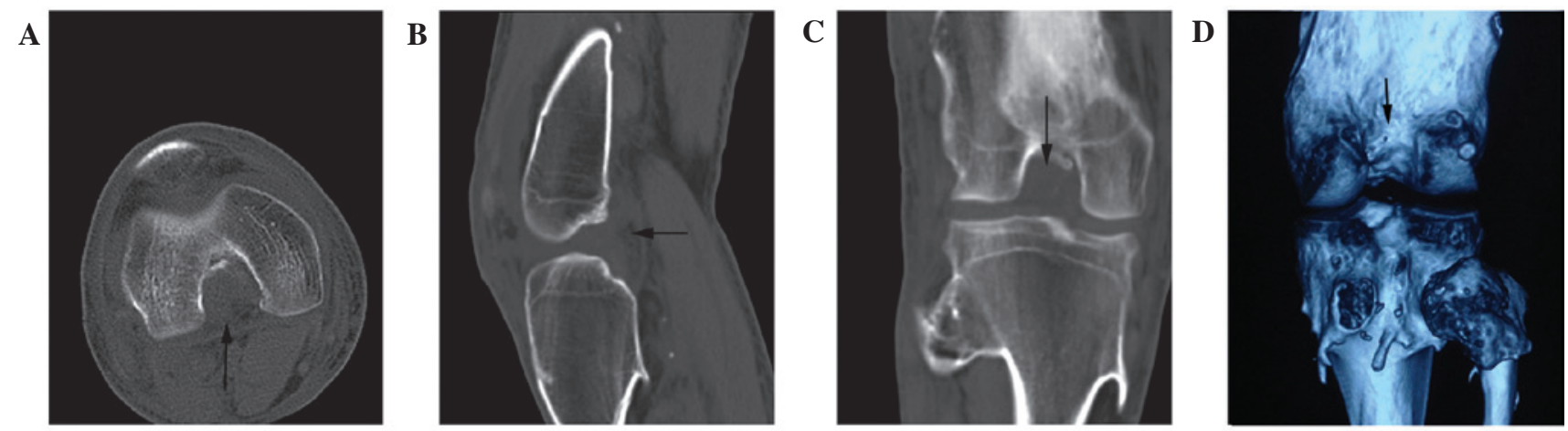

Figure 6. Postoperative (A-C) CT and (D) 3D CT reconstruction of the right knee. Arrows indicate the locations of the intra-articular masses preoperatively. Using the same scanning layers and positioning as in Fig. 1, the CT images in (A-C) demonstrate that the three masses had been removed and (D) the 3D CT reconstruction revealed a good level of intercondylar fossa space in the femur. CT, computed tomography.

In order to determine the genetic cause of HMO in this case, the patient and his mother underwent genetic analysis. The pedigree of this Chinese family is shown in Fig. 7A. All study procedures were approved by the Ethics Review Committee of the Zhejiang Provincial People's Hospital (Hangzhou, China) and were conducted following receipt of written informed consent from the patient and the patient's family. Genomic DNA was purified from peripheral blood leukocytes using an Axygen ${ }^{\circledR}$ AxyPrep Nucleic Acid Purification kit (Corning Life Sciences, Union City, CA, USA), according to the manufacturer's instructions. The Sanger method of direct DNA sequencing was performed on the EXT1 and EXT2 coding regions, including the 100-bp flanking intron-exon junctions. This was achieved by performing polymerase chain reactions of genomic DNA followed by sequencing reactions with Sanger sequencing chemistry, using the BigDye ${ }^{\circledR}$ Terminator v3.1 Cycle Sequencing kit on a 3730XL automated sequencer (Applied Biosystems Inc., Foster City, CA, USA). The primer sequences used are those previously cited by Cao et al (11). Sequencing data were analyzed by Sequencer Demo 3.0 and Mutation Surveyor ${ }^{\circledR}$ Demo software (version 4.0; SoftGenetics, LLC, State College, PA, USA) using reference sequences from the National Center for Biotechnology Information (NM_000127.2 for EXT1; NM_001178083.1 for EXT2).

A heterozygous mutation was identified in the genomic DNA of the patient. A substitution in exon 1 of the EXT1 gene (c.115G $>$ T) changed a leucine codon into a stop codon (p.E39X) in the EXT1 protein (Fig. 7B and C; Table I). The patient was also heterozygous for the synonymous mutation in exon 9 of the EXT1 gene (Table I). The same heterozygous exon 1 (c.115G>T; p.E39X) nonsense mutation and the synonymous mutation were detected in the patient's 
Table I. EXT1 gene changes detected by polymerase chain reaction and direct sequencing.

\begin{tabular}{lcccccc}
\hline ID & Gene name & Location & Nucleotide change & Amino acid change & Mutation type & Category \\
\hline II2 $^{\text {a }}$, III $1^{\text {b }}$ & EXT1 & Exon 1 & $115 \mathrm{G}>$ T(het) & $39 \mathrm{E}>\mathrm{X}$ & Nonsense & SNV \\
II2, III1 & EXT1 & Exon 9 & $1761 \mathrm{C}>$ T(het) & $587 \mathrm{E}>\mathrm{E}$ & Synonymous & SNV \\
\hline
\end{tabular}

apatient's mother; b patient. het, heterozygous; EXT1, exostosin-1; SNV, single nucleotide variant.
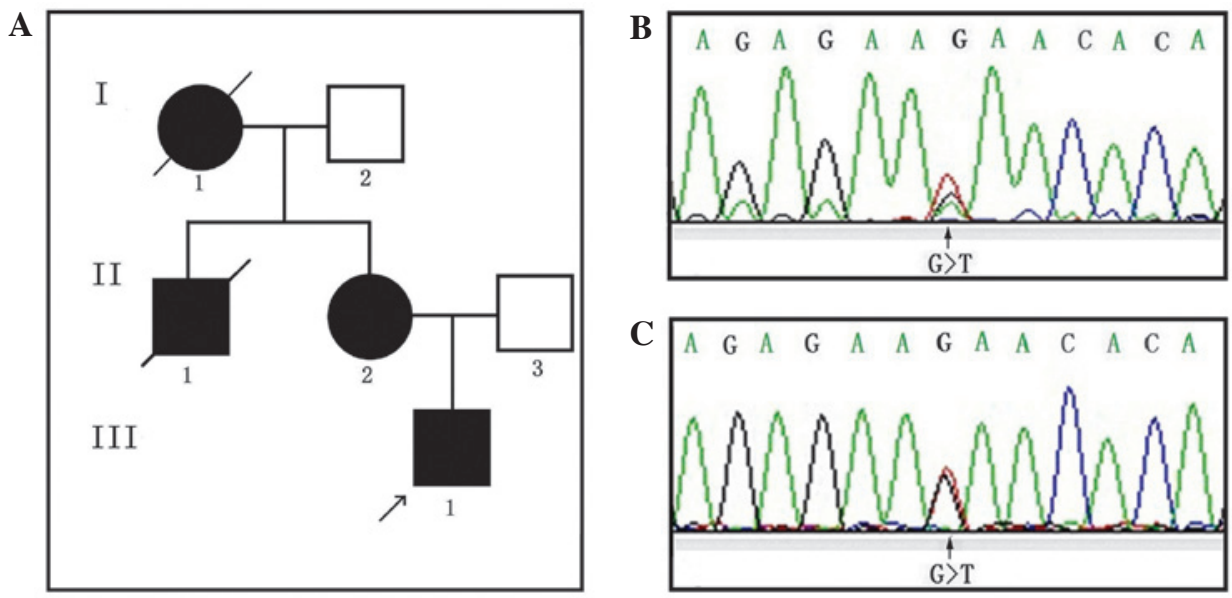

Figure 7. (A) Pedigree of the family with hereditary multiple osteochondromas. There was no consanguinity in this family. Filled symbols represent affected individuals and empty symbols indicate unaffected family members. Circles and squares indicate women and men, respectively. The current patient is represented by the proband (III1; arrow). Gene analysis, showing the exon 1 sequence of the EXT1 gene of (B) the patient and (C) the patient's mother. The arrow indicates the precise mutation observed in the patient (c. 115G >T). EXT1, exostosin-1.

mother. c.115G $>$ T; p.E39X has previously been reported as a disease-causing mutation (12). However, to the best of our knowledge, the current study is the first report this mutation in a Chinese individual.

\section{Discussion}

Intra-articular osteochondromas are rare in patients with HMO and have, thus far, only been described as case reports in the literature. Patients with HMO exhibit osteochondromas in the intra-articular region of the hip and ankle joints, causing functional joint defects in certain cases (13-15). It is theoretically feasible that an osteochondroma could remain located intra-articularly within the hip and ankle joints, as the neck of the femur and the talus are located in these joints. The knee is also a common location for intra-articular osteochondromas due to the large size of the joint capsule. However, in the context of HMO, intra-articular osteochondromas of the knee joint have rarely been described. To the best of our knowledge, only two cases of intra-articular osteochondromas in patients with HMO have been described in the literature. Takahashi et al (8) reported the first case of a patient exhibiting HMO with an osteochondroma in the knee joint. The mass was located in the anterolateral site of the distal femur, impinging upon the lateral edge of the lateral facets of the patella and inducing an inflammatory response in the lateral capsule of the patellofemoral joint. The second case of an osteochondroma within the knee joint was reported by Matsumoto et al (16). In this case, the osteochondroma was small and manifested as a loose body measuring $\sim 1 \mathrm{x} 1 \mathrm{x} 2 \mathrm{~cm}$. The lesions was located posterior to the PCL, causing pain and limited joint function. However, the present case was distinct in a number of ways from the previous two cases. Firstly, the current study reported a greater number of intra-articular osteochondromas that were also larger in size. Secondly, the intra-articular and extra-articular osteochondromas were resected and pathologically compared. Thirdly, HMO was clinically diagnosed in the current patient, and was also genetically diagnosed via the identification of a nonsense mutation in the EXT1 gene.

Intra-articular osteochondromas are difficult to visualize and are only detectable through imaging or arthroscopic exploration. In certain cases, even the use of plain radiographs cannot provide a definitive diagnosis. CT scans provide a clear image of the bony structure of the joint, as well as the outline of bony masses within it. Furthermore, MRI imaging aids in determining the nature of the mass and clearly delineates adjacent tissues, including ligaments and tendons. Symptoms of intra-articular osteochondromas may result from exostotic impingement on adjacent intra-articular structures. In the current case study, plain radiographs identified the lesion at the intercondylar fossa. In addition, a CT scan revealed three intra-articular masses, and MRI was utilised to further characterise the nature of the masses and to visualise the adjacent soft structures. The first mass was located adjacent to the PCL; exerting pressure on the ligament (Fig. 3).Furthermore, the area where the three bony, loose bodies were located impacted the posterior contour of the medial condyle of the tibia upon flexion (Fig. 2D). 
Therefore, the patient experienced pain during loaded flexion, as well as a significant decrease in bending range.

Surgical excision is the only reliable treatment strategy for symptomatic intra-articular osteochondromas. It is performed in order to treat the patient and as a means of making a definitive diagnosis. Surgical excision may be performed using an open technique or arthroscopically. Arthroscopy is minimally invasive, does not cause significant trauma to the musculature, and is useful for treating meniscal tears and synovitis, as well as removing loose bodies from the joint. The treatment of intra-articular osteochondroma with precise extension using arthroscopic techniques has recently been reviewed in a number of studies and has demonstrated good overall results $(8,16,17)$. The advantages of arthroscopic resection of osteochondromas include a cosmetically pleasing result, a potentially less painful procedure, tailored resection of the lesion and a low complication rate. This technique also results in rapid recovery (17).

The origin and pathogenesis of the intra-articular loose osteochondromas presented in the current study are controversial. In specific cases of extraskeletal osteochondromas, the origin of intra-articular osteochondromas have been shown to be soft tissues $(18,19)$. Jaffe $(20)$ initially introduced the concept of para-articular osteochondromas in 1958, using the synonymous terms 'para-articular chondromas' and 'intracapsular chondromas' to describe osteochondral metaplasia occurring in the fibrous joint capsule or soft tissue adjacent to the joint. Subsequently, Milgram and Dunn (19) used the term 'para-articular osteochondroma' to differentiate between intra-articular osteochondroma and synovial osteochondromatosis. Synovial osteochondromatosis typically includes multiple osteochondrous synovium nodules with loose bodies in the joint (21); the multiple lobules of proliferative cartilage originate in the synovial joint tissue. By contrast, para-articular osteochondromas originate from a cartilaginous metaplasia of the articular and para-articular connective tissues (22). They are typically composed of a single large calcified mass of multiple osteochondral nodules, with no histological evidence of a synovial origin (23). The two aforementioned osteochondromas originate from intra-articular soft tissues and are therefore not true osteochondromas, which originate in the growth plates of long bones.

However, the patient in the present case may not represent either of these types of osteochondromas. The current patient was clinically and genetically diagnosed with HMO, and histological analysis of the intra-articular osteochondroma did not reveal any evidence of synovial or other soft tissue origin. Additionally, histological analysis demonstrated that the intra-articular osteochondroma appeared to have a thinner cartilage cap. This indicates that the osteochrondroma may have stopped growing for a long time period, as the thickness of the cartilage cap is positively correlated with the growth activity of the osteochondroma and growth rate decreases with age (24). Once osteochondromas stop growing, the cartilage cap shrinks due to compression by adjacent structures. Therefore, it is proposed that the osteochondroma in the case described, originated at the posterior end-plate of the femur or tibia a number of years prior to presentation and grew towards the articular cavity of the knee. The pedicle may subsequently have torn, allowing the osteochondroma to become loose in the knee joint. At that point, growth of the osteochondroma may have stopped. As the mass remained in the intercondylar fossa of the knee and was compressed by the bony structure of the intercondylar fossa, the cartilage cap may have begun to degenerate. Exposure to the synovial fluid and movement of the knee over time may have caused a single loose osteochondroma to divide into three distinct masses. However, the aetiology of the intra-articular osteochondromas described in the present study, as well as the histological differences between intra-articular and extra-articular osteochondromas in the context of HMO, remains unknown and requires further investigation.

\section{Acknowledgements}

The authors would like to thank the patient and his family members for their interest and cooperation. The present study was supported by a grant, awarded to Professor Qing Bi, by the Natural Science Foundation of Zhejiang Province (grant no. Y2100731) and the Medical Science and Technology Project in Zhejiang Province (grant no. Y201024964).

\section{References}

1. Stieber JR and Dormans JP: Manifestations of hereditary multiple exostoses. J Am Acad Orthop Surg 13: 110-120, 2005.

2. Solomon L: Hereditary Multiple Exostosis. Am J Hum Genet 16: 351-363, 1964.

3. Cook A, Raskind W, Blanton SH, Pauli RM, Gregg RG, Francomano CA, Puffenberger E, Conrad EU, Schmale G, Schellenberg G, et al: Genetic heterogeneity in families with hereditary multiple exostoses. Am J Hum Genet 53: 71-79, 1993.

4. Wu YQ, Heutink P, de Vries BB, Sandkuijl LA, van den Ouweland AM, Niermeijer MF, Galjaard H, Reyniers E, Willems PJ and Halley DJ: Assignment of a second locus for multiple exostoses to the pericentromeric region of chromosome 11. Hum Mol Genet 3: 167-171, 1994.

5. Le Merrer M, Legeai-Mallet L, Jeannin PM, Horsthemke B Schinzel A, Plauchu H, Toutain A, Achard F, Munnich A and Maroteaux P: A gene for hereditary multiple exostoses maps to chromosome 19p. Hum Mol Genet 3: 717-722, 1994.

6. PorterDE,LonieL,FraserM,Dobson-StoneC,PorterJR,MonacoAP and Simpson AH: Severity of disease and risk of malignant change in hereditary multiple exostoses. A genotype-phenotype study. J Bone Joint Surg Br 86: 1041-1046, 2004.

7. Cao L, Liu F, Kong M, Fang Y, Gu H, Chen Y, Zhao C, Zhang S and Bi Q: Novel EXT1 mutation identified in a pedigree with hereditary multiple exostoses. Oncol Rep 31: 713-718, 2014.

8. Takahashi M, Nishihara A, Ohishi T, Shiga K, Yamamoto K and Nagano A: Arthroscopic resection of an intra-articular osteochondroma of the knee in the patient with multiple osteochondromatosis. Arthroscopy 20 (Suppl 2): 28-31, 2004.

9. Vanhoenacker FM, Van Hul W, Wuyts W, Willems PJ and De Schepper AM: Hereditary multiple exostoses: From genetics to clinical syndrome and complications. Eur J Radiol 40: 208-217, 2001.

10. Wicklund CL, Pauli RM, Johnston D and Hecht JT: Natural history study of hereditary multiple exostoses. Am J Med Genet 55: 43-46, 1995.

11. Cao L, Liu F, Kong M, Fang Y, Gu H, Chen Y, Zhao C, Zhang S and Bi Q: Novel EXT1 mutation identified in a pedigree with hereditary multiple exostoses. Oncol Rep 31: 713-718, 2014.

12. Alvarez CM, De Vera MA, Heslip TR and Casey B: Evaluation of the anatomic burden of patients with hereditary multiple exostoses. Clin Orthop Relat Res 462: 73-79, 2007.

13. Bonnomet F, Clavert P, Abidine FZ, Gicquel P, Clavert JM and Kempf JF: Hip arthroscopy in hereditary multiple exostoses: A new perspective of treatment. Arthroscopy 17: E40, 2001.

14. El-Fiky TA, Chow W, Li YH and To M: Hereditary multiple exostoses of the hip. J Orthop Surg (Hong Kong) 17: 161-165, 2009.

15. Rook FR: Intra-articular osteochondroma of the astragalus. Am J Surg 85: 807-810, 1953. 
16. Matsumoto Y, Matsuda S, Matono K, Oda Y, Tsuneyoshi M and Iwamoto Y: Intra-articular osteochondroma of the knee joint in a patient with hereditary multiple osteochondromatosis. Fukuoka Igaku Zasshi 98: 425-430, 2007.

17. Schmoyer S and Ciullo JV: Arthroscopic resection of an osteochondroma of the knee. Arthroscopy 17: 765-767, 2001.

18. Das AK and Mukherjee DR: Giant osteochondral loose body of the knee joint. A case report. J Bone Joint Surg Am 60: 559-560, 1978

19. Milgram JW and Dunn EJ: Para-articular chondromas and osteochondromas: A report of three cases. Clin Orthop Relat Res 148: 147-151, 1980.

20. Jaffe HL: Tumors and tumorous conditions of the bones and joints. Acad Med 34: 72, 1959.
21. Schofield TD, Pitcher JD and Youngberg R: Synovial chondromatosis simulating neoplastic degeneration of osteochondroma: Findings on MRI and CT. Skeletal Radiol 23: 99-102, 1994.

22. Rizzello G, Franceschi F, Meloni MC, Cristi E, Barnaba SA, Rabitti C and Denaro V: Para-articular osteochondroma of the knee. Arthroscopy 23: 910.e1-910.e4, 2007.

23. Karlin CA,DeSmet AA, Neff J,LinF, Horton W and Wertzberger JJ: The variable manifestations of extraarticular synovial chondromatosis. AJR Am J Roentgenol 137: 731-735, 1981.

24. Hudson TM, Springfield DS, Spanier SS, Enneking WF and Hamlin DJ: Benign exostoses and exostotic chondrosarcomas: Evaluation of cartilage thickness by CT. Radiology 152: 595-599, 1984. 